\title{
Diversidade entre linhagens e importância de caracteres relacionados à longevidade em vaso de linhagens de pimenteiras ornamentais ${ }^{(1)}$
}

\author{
ELIZANILDA RAMALHO DO RÊGO(2,8); DAMIANA FERREIRA DA SILVA ${ }^{(4)}$; MAILSON MONTEIRO DO RÊGO(3,8); \\ RUSTHON MAGNO CORTEZ DOS SANTOS ${ }^{(5)}$; MORYB JORGE DA LIMA COSTA SAPUCAY(6) E DENIZIA RIBEIRO DA SILVA $^{(7)}$
}

\begin{abstract}
RESUMO
Apesar de sua reconhecida importância econômica e social, a cultura da pimenta ainda é pouco estudada. O objetivo do presente trabalho foi avaliar a longevidade de linhagens de pimentas pertencentes ao gênero Capsicum, quanto à vida útil em vaso e sua variabilidade. $\mathrm{O}$ delineamento experimental utilizado foi inteiramente casualizado com três repetições e seis tratamentos (linhagens $76,82,72,132,83$ e 134). Após a frutificação e maturação dos frutos as plantas foram expostas a condições simuladas de transporte e posteriormente transferidas para uma sala, foram avaliadas diariamente quanto à capacidade de envelhecimento em vaso. As características avaliadas estatisticamente foram o número de folhas caídas, número de folhas amarelas, número de folhas murchas, número de frutos caídas e longevidade em vaso. Os dados obtidos foram submetidos à análise de variância, com posterior separação das médias pelo teste Duncan $(\mathrm{p} \leq 0.05)$. A variabilidade genética foi acessada com base na distância generalizada de Mahalanobis com posterior agrupamento pelo método de Tocher. A importância relativa das características foi calculada baseando-se no método proposto por SINGH. A linhagem 132 foi aquela que apresentou o maior número de folhas murchas e as linhagens 83 e 72 os menores números. A linhagem 82 foi aquela que apresentou o maior número de frutos caídos, e para a longevidade destacouse as linhagens $82,132,83$ e 134. Utilizando o método de Tocher agrupou-se no primeiro grupo as linhagens 132,143 e 75 , no segundo 82 e 72, e 83. Pelo método de SINGH a variável que mais contribuiu com a divergência foi o número de folhas caídas com $43 \%$ e a que menos contribuiu foi o número de folhas amarelas com $2 \%$.
\end{abstract}

Palavras-chaves: Capsicum, plantas ornamentais, vida de vaso.

\section{Diversity among lineages and relative importance of longevity traits in potted ornamental peppers}

The chilli peppers an important vegetable crop in Brazil. Although there are little studies with the Capsicum species. The aim of this work was to evaluate the pot life longevity of chilli lineages and their variability. The experimental design was performed in an entirely random design with three replicates and six treatments (lineages: 76, 82, 72, 132, 83 and 134). After the fructification and fruit maturation the plants were submitted to simulated transport conditions and later transferred to room temperature. The pot aging ability was daily evaluated. The traits observed were number of dropped leaves, number of yellow leaves, number of dry leaves, number of dropped fruits and longevity. The data were submitted to Analysis of variance and the means are separated by Duncan's test $(\mathrm{p} \leq 0.05)$. The genetic variability was done by Mahalanobis distance with grouping by Tocher's method. The relative importance of traits to variability was done by SINGH's method. The lineage 132 presented major number of leaves dropped and the lineages 83 and 72 the minor values for this trait. The major numbers of fruit dropped was presented by lineage 82 . The lineages that had survived for longer were 82, 132, 83 and 134. The Tocher's method formed two groups: the first one with lineages 132, 143 and 75; and the second one with 82, 72 and 83. The SINGH's method pointed out the variable number of yellow leaves ( $2 \%)$ and the trait that most contribute to diversity was the number of dropped leaves (43\%).

Keywords: Capsicum,ornamental plants, pot life.

\section{INTRODUÇÃo}

O Brasil produz hoje aproximadamente 900 milhões de unidades de flores e plantas ornamentais e o setor emprega cerca de oito mil produtores. Os principais estados produtores são Minas Gerais, Rio de Janeiro e São Paulo (RBHO, 2009), entretanto, ainda existem sérios entraves na fase de pós-produção que afetam a qualidade e a vida de vaso de plantas ornamentais em geral, sendo que a sensibilidade ao etileno é um dos mais importantes.

As espécies de pimentas pertencem ao gênero Capsicum, família Solanaceae e possuem aproximadamente 27 espécies. Dentre estas espécies, cinco são domesticadas e largamente cultivadas e utilizadas pelo homem, que são às seguintes: $C a p$ sicum annuum; C. bacccatum; C. chinense; $C$. frutescens e $C$. pubescens, sendo apenas $C$. pubescens não cultivada no Brasil (BOSLAND, 1996).

As pimentas foram possivelmente os primeiros aditivos alimentares utilizados pelas civilizações antigas do México e da América do Sul. Suas características atribuem aroma, cor e sabor aos alimentos, tornando-os mais atraentes. Além de estimulantes do apetite e auxiliares da digestão, os frutos de Capsicum são fontes importantes de três antioxidantes naturais: vitamina $\mathrm{C}$, carotenóides e vitamina $\mathrm{E}$ (GRAVINA et al, 2009; RÊGO et al., 2009b).

O Brasil conta com uma área de produção do gênero Capsicum estimada em 13.000 ha e produção anual de cerca de 280.000 toneladas, tanto para consumo fresco como processado, envolvendo recursos na ordem de 1,5 milhão de dólares somente na comercialização de sementes (REIFSCHNEIDER,

\footnotetext{
(1) Recebido para publicação em 17/10/2009 e aceito em 01/02/2011.

(2)UFPB, Professora do DCFS-CCA-UFPB.

(3) Professor do Departamento de Fitotecnia, CCA-UFPB.

(4) UFPB, Mestranda em Agronomia-CCA-UFPB -Bolsista CNPq.

(5) Mestrando em Genética e melhoramento-UFV, Bolsista CNPq.

(6) Mestrando em Fitotecnia-UFV, Bolsista CNPq.

(7) Bolsista Probex_CCA-UFPB.

${ }^{(8)}$ Bolsista de produtividade em pesquisa CNPq. Centro de Ciências Agrárias CCA-UFPB - Campus II, Areia-PB, 58397-000, e-mails: elizanilda@cca.ufpb.

br,damyagro@hotmail.com, mailson@cca.ufpb.br, rusthoncortez@hotmail.com,moryb_sapucay@hotmail.com.
} 
2000). Apesar de sua reconhecida importância econômica e social, a cultura da pimenta ainda é pouco estudada no Brasil, em todas suas fases do sistema de produção. A busca por melhor qualidade, preços e custos têm exigido dos produtores maior eficiência técnica e econômica na condução dos sistemas de produção.

Dentre as plantas ornamentais em vaso, as pimentas têm se destacado pela sua crescente e contínua aceitação pelo mercado consumidor. Em princípio qualquer espécie de pimenta poderia ser utilizada como planta ornamental, porém as espécies de menor porte são mais indicadas para o plantio em vasos, principalmente na decoração de ambientes internos (VIEIRA, 2002).

A crescente demanda de mercado, estimada em 80 milhões de reais ao ano, tem impulsionado o aumento da área cultivada e o estabelecimento de agroindústrias, tornando o agronegócio Capsicum um dos mais importantes do país (RIBEIRO et al., 2003). Surgindo assim a necessidade de estudos que visem o conhecimento da cultura e viabilizem metas como aumento da resistência a doenças, vida de vaso, tolerância a ambientes adversos e transporte dentre outros fatores bióticos e abióticos que influenciam a sua produção (OLIVEIRA et al., 2003; RÊGO, et. al., 2006). Diante do exposto o objetivo do presente trabalho foi avaliar a longevidade de linhagens de pimentas pertencentes ao gênero Capsicum, quanto à vida útil em vaso, bem como sua variabilidade genética.

\section{MATERIAL E MÉTODOS}

O trabalho foi conduzido no Centro de Ciências Agrária da Universidade Federal da Paraíba, localizado no município de Areia-PB na Microrregião do Brejo Paraibano, com altitude de $574,62 \mathrm{~m}$, latitude $6^{\circ} 58^{\prime} \mathrm{S}$, e longitude $35^{\circ} 42^{\prime} \mathrm{WGr}$, predomina na região clima quente e úmido com chuvas de outono-inverno. A temperatura média anual oscila entre 23 a $24^{\circ} \mathrm{C}$, com pequenas variações mensais.

O delineamento experimental utilizado foi inteiramente casualizado com três repetições e seis tratamentos, sendo os mesmos compostos pelas linhagens $76,82,72,132,83 \mathrm{e}$ 134. As linhagens escolhidas para a realização do experimento foram previamente selecionadas no Banco de Germoplasma do Centro de Ciências Agrárias-UFPB (RÊGO et al, 2009b), considerando-se a arquitetura da planta, hábito de crescimento, tamanho do fruto, forma do fruto, tamanho da folha, entre outras características que caracterizam as plantas ornamentais.

As sementes das pimentas foram inicialmente semeadas em tubetes contendo substrato comercial. Após um mês a partir da emergência foram transplantadas para vasos de polietileno contendo $700 \mathrm{~mL}$ com substrato comercial. Após a frutificação e maturação dos frutos as plantas foram expostas a condições simuladas de transporte por um período de $48 \mathrm{~h}$, onde não foram irrigadas e não estavam expostas a luz solar, após esse período foram transferidas para uma sala com $12 \mathrm{~h}$ de luz e irrigadas quando necessário.

As pimenteiras foram avaliadas diariamente quanto à capacidade de envelhecimento em vaso onde foram analisados os parâmetros abscisão e perda de turgescência das folhas e dos frutos, baseados em uma escala descritiva da qualidade como segue: estádio 0- excelente turgidez e rigidez das folhas e frutos com cor viva e brilhante, folhas com cor verde e brilhante; estádio 1- boa turgidez e rigidez das folhas e frutos, boa coloração dos frutos; estádio 2- boa turgidez dos frutos, início do murchamento ou clorose foliar e descoloração dos frutos; estádio 3- murchamento dos frutos, clorose foliar e abscisão. O estádio 3 define o término da longevidade da planta, quando houve perda do valor ornamental e comercial: amareladas e com frutos murchos ou mortas. A partir do estádio 2 as plantas já não podem ser comercializadas.

As características avaliadas estatisticamente foram o número de folhas caídas, o número de folhas amarelas, o número de folhas murchas, o número de frutos que caíram e a longevidade em vaso. $\mathrm{O}$ experimento que foi conduzido de 05 de setembro a 20 de novembro de 2008. Os dados obtidos das diferentes características foram submetidos à análise de variância, obedecendo ao delineamento inteiramente casualizado com posterior separação das médias pelo teste Duncan, em nível de 5\% de probabilidade. A variabilidade genética foi acessada com base na distância generalizada de Mahalanobis com posterior agrupamento pelo método de Tocher. A importância relativa das características para a divergência foi calculada baseando-se no método proposto por SINGH (1981). As análises estatísticas foram realizadas por meio do o programa computacional GENES (CRUZ, 2001).

\section{RESULTADOS E DISCUSSÃO}

Houve diferença significativa entre as médias das linhagens em nível de $5 \%$ de probabilidade pelo teste $\mathrm{F}$, para todas as características estudadas, exceto para o número de folhas caídas e o número de folhas amarelas (tabela 1). Já para o número de folhas murchas, número de frutos caídos e longevidade houve diferenças entre as médias ao serem comparadas pelo teste Duncan, sendo que a linhagem 132 foi aquela que apresentou o maior número de folhas murchas e as linhagens 83 e 72 as que apresentaram os menores números, respectivamente. A linhagem 82 foi aquela que apresentou o maior número de frutos caídos, e para a duração as linhagens que persistiram por um maior número de dias foram as $82,132,83$ e 134 , e as linhagens 76 e 72 foram as que duraram menos dias durante a condução do experimento (Tabela 2). RÊGO et al. (2009a), em trabalho demonstraram que as linhagens 137, 144 e 146 apresentaram a maior longevidade em vaso que duraram por aproximadamente 36 dias.

Valores altos de CV, maiores que $62 \%$ foram encontrados para as características número de folhas caídas, número de folhas amarelas e número de folhas murchas, sendo que o alto valor de CV $(62,01)$, desta última característica, não impediu a detecção da variabilidade na mesma (tabela 1). SILVA et al (2011) em seu trabalho com pimenteiras determinou que os valores de $\mathrm{CV}$ variam de acordo com a característica, com o acesso e com a espécie. Neste mesmo trabalho, os autores demonstraram que em um determinado acesso de Capsicum frutescens o $\mathrm{CV}$ para espessura do pericarpo, por exemplo, foi considerado muito alto quando apresentou valores maiores que $28 \%$, já em outro acesso, da mesma espécie, o CV só foi considerado muito alto quando atingiu valores maiores que $48 \%$. Este mesmo comportamento pode estar ocorrendo para as variáveis relacionadas com a pós-produção em pimenteiras ornamentais.

As linhagens formaram 03 grupos: no primeiro grupo encontram-se as linhagens 132, 134 e 76, o segundo grupo foi formado pelas linhagens 82 e 72 , e o grupo três foi formado apenas pela linhagem 83 (Tabela 3). O uso de linhagens divergentes no programa de melhoramento pode geração F1 com maior heterose (RÊGO et al, 2009b). Uma vez que as linhagens 82, 83, 132 e 134 apresentaram maior vida de vaso e 
pertencem a diferentes grupos, as mesmas podem ser cruzadas para obtenção de F1 e exploração da heterose híbrida.

Pelo método de SINGH (1981), utilizado para avaliar a importância relativa de 5 características quantitativas, determinou-se que três destas características contribuíram com 83 $\%$ da divergência genética, enquanto duas contribuíram com apenas $17 \%$ (Figura 1). A variável que mais contribuiu com a divergência foi o número de folhas que caíram com $43 \%$ e a que menos contribuiu foi o número de folhas amarelas com $2 \%$, seguida da duração com $15 \%$. Estas variáveis podem ser descartadas em futuros estudos de divergência conforme RÊGO et al. (2003). Estes mesmos autores, ao estudarem o gênero Capsicum verificaram considerável variabilidade dentro do gênero, detectada tento pelo método de Tocher como pelo métodos dos componentes principais.

\section{REFERÊNCIAS}

BOSLAND, P.W. Capsicums: Innovative uses of an ancient crop. In: J. Janick (ed.), Progress in new crops. ASHS Press: Arlington, 1996, p. 479-487.

CRUZ C.D. Aplicativo computacional em genética e estatística. Viçosa: UFV, 2001. 648 p.

GRAVINA, O., HENZ, G.P., CARVALHO, S.I. C. Conservação pós-colheita de pimentas da espécie Capsicum chinense com filme de PVC em duas temperaturas. Disponível em: http://www.abhorticultura.com.br/biblioteca/arquivos/Download/Biblioteca/44_159.pdf. Acessado em: 10/04/2009.

OLIVEIRA, J.G., CHIQUIERE, T.B., OLIVEIRA JÚNIOR, L.F.G., BASTOS, P.A., BRESSAN-SMITH, R. Resposta ao Estresse Hídrico em alguns Cultivares de Capsicum spp. In: CONGRESSO BRASILEIRO DE MELHORAMENTO DE PLANTAS, 2., Porto Seguro-BA, 2003. Anais... 2003.

RBHO, Revista Brasileira de Horticultura Ornamental - http://www.sbfpo.com.br/noticias/ acesso em 14 de março, 2009.

RÊGO, E.R. do, RÊGO, M.M., CRUZ, C.D., CECON, P.R., AMARAL, D.S.S.L., FINGER, F. Genetic diversity analysis of peppers: a comparison of discarding variable methods. Crop Breeding and Applied Biotechnology, Viçosa, v. 3, n.
1, p. 19-26, 2003.

RÊGO, E. R., FINGER, F.L, CRUZ, C. D., RÊGO, M.M. Caracterização, diversidade e estimação de parâmetros genéticos em pimenteiras (Capsicum spp.). In: Encontro Nacional do Agronegócio Pimentas (Capsicum spp.), 2., Brasília, 2006. Anais... 2006.

REGO, E. R., RÊGO, M. M., SILVA, D. F., CORTEZ, R. M., SAPUCAY, M. J. L. C., SILVA, D. R., SILVA JUNIOR, S. J. Selection for leaf and plant size and longevity of ornamental peppers (Capsicum spp.) grown in greenhouse condition. Acta Horticulturae, v. 829, p. 371-375, 2009a.

RÊGO, E.R., RÊGO, M.M., FINGER, F.L., CRUZ, C.D., CASALI, V.W.D. A diallel study of yield components and fruit quality in chilli peppers (Capsicum baccatum). Euphytica,v. 168, 275-287, $2009 \mathrm{~b}$.

REIFSCHNEIDER, F.J.B. Capsicum: pimentas e pimentões no Brasil. Brasília: Embrapa Comunicação Transferência de Tecnologia, 2000, 113 p.

RIBEIRO, C.S.C., SOUZA, O.B., LOPES, D., REIFSCHNEIDER, F.B. Programa de Melhoramento Genético de Capsicum da Embrapa Hortaliças para Processamento Industrial. In $2^{\circ}$ CONGRESSO BRASILEIRO DE MELHORAMENTO DE PLANTAS, In: Congresso Brasileiro de Melhoramento de Plantas, Centro, 2., Porto Seguro-BA, 2003. Anais... 2003.

SILVA, A.R., CECON, P.R., RÊGO, E.R., NASCIMENTO M. Avaliação do coeficiente de variação experimental para caracteres de frutos de pimenteiras, Revista Ceres,v. 58, n. 2, 168$171,2011$.

SINGH, D. The relative importance of characters affecting genetic divergence. The Indian Journal of Genetic and Plant Breeding, New Delhi, v. 41, p. 237-245, 1981.

VIEIRA, M. A . 2002. Uso de polímero hidroabsorvente efeitos sobre a qualidade de substratos hortícolas e crescimento de mudas de pimentão ornamental. Pelotas: Faculdade de Agronomia Eliseu Maciel, UFPel, 2002. 113p. Tese (Doutorado em Agronomia - Produção Vegetal).

Tabela 1. Resumo da análise de variância em 06 linhagens de pimenta. (Número de folhas que caíram - $\mathrm{N}^{\mathrm{o}} \mathrm{FC}$; Número de folhas amarelas- No FA; Número de folhas murchas- $N^{\circ}$ FM; Número de frutos que caíram- No FRC; Duração- DUR). Areia, CCA, 2008

Table 1. Analysis of variance of six chilli lineages. Number of leaves dropped $-N^{o} F C$; number of yellow leaves- $N^{\circ}$ FA; number of dry leaves- $N^{o}$ FM; number of dropped fruits- $N^{o}$ FRC; longevity- DUR). Areia, CCA, 2008

\begin{tabular}{|c|c|c|c|c|c|}
\hline \multirow{2}{*}{ Fonte de Variação } & \multicolumn{5}{|c|}{ Quadrados Médios } \\
\hline & $\mathrm{N}^{\mathrm{o}} \mathrm{FC}$ & $\mathrm{N}^{\mathrm{o}} \mathrm{FA}$ & $\mathrm{N}^{\mathrm{o}} \mathrm{FM}$ & $\mathrm{N}^{\mathrm{o}} \mathrm{FRC}$ & DUR \\
\hline Tratamentos & $1232,36 \mathrm{~ns}$ & $574.48 \mathrm{~ns}$ & $2692610,45^{*}$ & $3,38 *$ & $864,13^{*}$ \\
\hline Resíduo & 1812,88 & 660.61 & 574524,83 & 0,74 & 177,61 \\
\hline C.V. $(\%)$ & 62,76 & 152.18 & 62,01 & 54,02 & 21,72 \\
\hline
\end{tabular}

ns e * = Não significativo e significativo a $5 \%$ de probabilidade, respectivamente, pelo teste $\mathrm{F}$. 
Tabela 2. Número de folhas que caíram - $\mathrm{N}^{\mathrm{o}} \mathrm{FC}$; Número de folhas amarelas- $\mathrm{N}^{\mathrm{o}} \mathrm{FA}$; Número de folhas murchas- $\mathrm{N}^{\mathrm{o}}$ FM; Número de frutos que caíram- $\mathrm{N}^{\circ} \mathrm{FRC}$; Duração- DUR de linhagens de pimenta

Table 2. Number of leaves dropped $-N^{o} F C$; number of yellow leaves- $N^{o} F A$; number of dry leaves- $N^{o}$ FM; number of dropped fruits- $N^{o}$ FRC; longevity-DUR of chilli lineages) Areia, CCA, 2008

\begin{tabular}{|c|c|c|c|c|c|}
\hline \multirow{2}{*}{ Linhagens } & \multicolumn{5}{|c|}{ Médias } \\
\hline & $\mathbf{N}^{\mathbf{0}} \mathbf{F C}$ & $\mathbf{N}^{0} \mathbf{F A}$ & $\mathbf{N}^{\mathbf{0}} \mathbf{F M}$ & $\mathbf{N}^{0}$ FRC & DUR \\
\hline 76 & $78,33 a$ & $8,33 a$ & $1781,00 \mathrm{ab}$ & $0,87 \mathrm{~b}$ & $37,66 \mathrm{~b}$ \\
\hline 82 & $50,66 a$ & $13,33 a$ & $548,66 \mathrm{bc}$ & $3,52 \mathrm{a}$ & $74,00 a$ \\
\hline 72 & $35,33 a$ & $11,00 a$ & $339,00 \mathrm{c}$ & $2,06 \mathrm{ab}$ & $42,00 \mathrm{~b}$ \\
\hline 132 & $87,66 a$ & $29,66 a$ & $2492,33 a$ & $0,99 \mathrm{~b}$ & $74,00 a$ \\
\hline 83 & $80,00 a$ & $37,66 a$ & $283,00 \mathrm{c}$ & $0,70 \mathrm{~b}$ & $74,00 a$ \\
\hline 134 & $75,00 a$ & $1,33 a$ & $1890,33 \mathrm{ab}$ & $1,42 \mathrm{~b}$ & $66,33 a$ \\
\hline
\end{tabular}

Médias seguidas pela mesma letra, em cada coluna, não diferem estaticamente entre si, de acordo com o teste de Duncan ( $\mathrm{p} \leq 0.05$ ).

Tabela 3. Agrupamento de 06 linhagens de pimenteiras pelo método de Tocher baseada na distância generalizadas de Mahalanobis. Areia, CCA, 2008.

Table 3. Grouping for six chilli lineages by Tocher's method based on Mahalanobis distance

\begin{tabular}{cc} 
Grupos & Linhagens \\
\hline 1 & $132,134,76$ \\
2 & 82,72 \\
3 & 83 \\
\hline
\end{tabular}

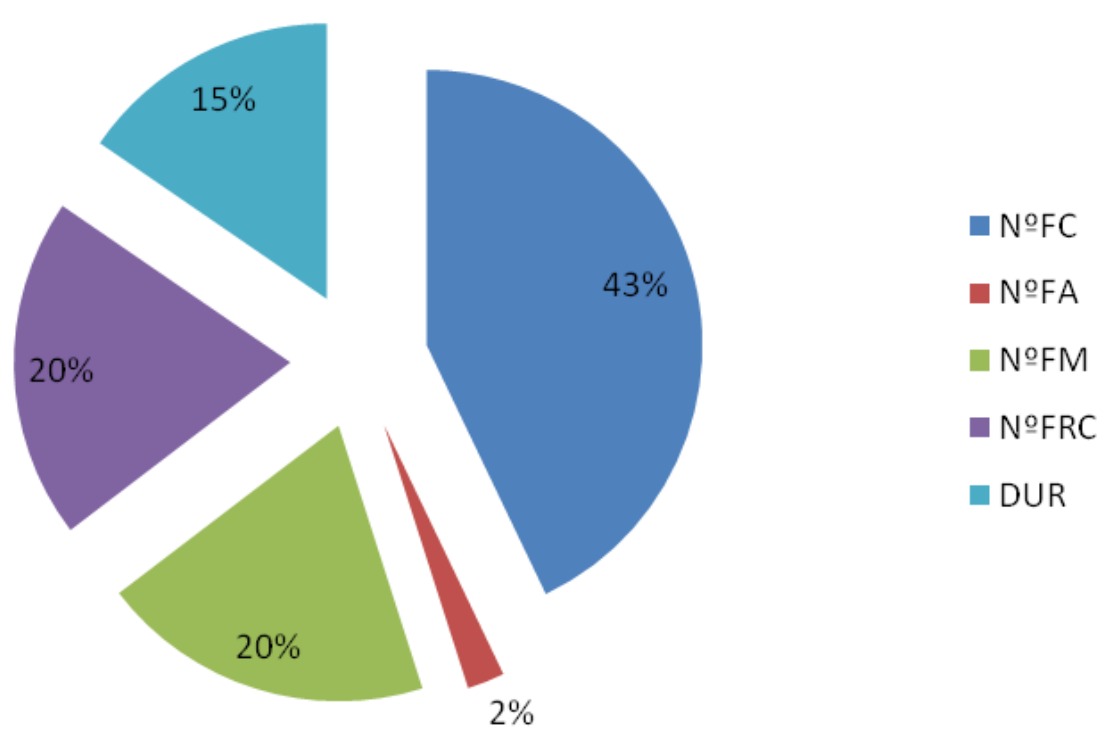

Figura 1. Contribuição relativa das características avaliadas (\%) para a divergência genética em pimenteira (Número de folhas que caíram - $\mathrm{N}^{\mathrm{o}}$ FC; Número de folhas amarelas- $\mathrm{N}^{\circ}$ FA; Número de folhas murchas- $\mathrm{N}^{\mathrm{o}}$ FM; Número de frutos que caíram- No FRC; Duração- DUR). Areia, CCA, 2008.

Figure 1. Evaluated traits relative importance (\%) for genetic diversity in chilli peppers. (Number of leaves dropped - $N^{o}$ FC; number of yellow leaves- $N^{o} F A$; number of dry leaves- $N^{o} F M$; number of dropped fruits- $N^{o} F R C$; longevity- DUR). 